\title{
Características clínicas, ecográficas y citohistológicas de nódulos tiroideos con sospecha de malignidad en un servicio de endocrinología de un hospital público
}

\section{Clinical, ultrasound and cytohystological features of thyroid nodules with suspicion of malignancy in a public hospital's endocrinology service}

\author{
Liz Kathia Mendoza-Montoya,' Socorro del Pilar Cornejo-Arenas,' \\ José Enrique Solis-Villanueva, ${ }^{2}$ Freddy Edmundo García-Ramos' y \\ María de los Ángeles Lazo-Porras ${ }^{3}$
}

\begin{abstract}
Mendoza-Montoya LK, Cornejo-Arenas SP, SolísVillanueva JE, García-Ramos FE, Lazo-Porras MA. Características clínicas, ecográficas y citohistológicas de nódulos tiroideos con sospecha de malignidad en un servicio de endocrinología de un hospital público. Rev Soc Peru Med Interna. 2019;32(2):48-53.
\end{abstract}

\begin{abstract}
RESUMEN
InTRODUcción. La importancia del estudio ecográfico y citológico del nódulo tiroideo radica en la identificación oportuna del cáncer tiroideo. Objetivo: Describir las características clínicas, ecográficas y cito-histológico de los nódulos tiroideos con sospecha de malignidad en pacientes atendidos en el área de ecografía del servicio de Endocrinología de un hospital público. Material y métodos: Se realizó un estudio prospectivo y descriptivo de los pacientes con nódulos tiroideos con sospecha de malignidad que acudieron al área de ecografía del servicio de Endocrinología del Hospital Nacional Arzobispo Loayza, Lima, durante el periodo marzo de 2016 a marzo de 2017. Los pacientes con nódulos tiroideos con criterios ecográficos (TI-RADS 4,5 y 6) y citológicos de malignidad (Bethesda IV, V y VI) fueron referidos al servicio de cirugía de cabeza y cuello. Un total de 27 pacientes completaron su estudio clínico, ecográfico y cito-histológico. Resultados: De los 27 pacientes tiroidectomizados, el 70,37\% presentó una neoplasia maligna de tiroides, siendo la mayoría pacientes mujeres mayores de 40 años de edad. La neoplasia más frecuente fue el carcinoma papilar de tiroides, el 68,42\% de nódulos fueron mayores a $10 \mathrm{~mm}$. La mayoría de pacientes con nódulos TI-RADS 4-5 y Bethesda IV, V y VI fue informada con histología como neoplasia maligna de tiroides; el 75\% de estos pacientes fue eutiroideo. Conclusión: La correcta categorización de los nódulos según sus características ecográficas (Clasificación TI-RADS) permite la identificación de los pacientes que requerirán oportunamente una biopsia. En este estudio se encontró que la mayoría de pacientes con neoplasia maligna de tiroides correspondían a nódulos $\geq 10 \mathrm{~mm}$ con criterios ecográficos TI-RADS 4b y 5, y criterios citológicos Bethesda V y VI, en su mayoría eutiroideos. Se afirma al carcinoma papilar como la neoplasia tiroidea más frecuente.
\end{abstract}

Palabras clave. Nódulo tiroideo, ultrasonido, citología, Bethesda, cáncer, tiroides.

\begin{abstract}
Introduction: The importance of echographic and cytological studies of the thyroid nodule lies in the timely identification of thyroid cancer. Objective: Describe the clinical, ultrasonographic and cyto-histological characteristics of thyroid nodules with suspicion of malignancy in patients treated in the ultrasound area of the Endocrinology service of a public hospital. Material and methods: A prospective and descriptive study of patients with thyroid nodules suspected of malignancy was conducted in the ultrasound area of the Endocrinology service of the Hospital Nacional Arzobispo Loayza during the period March 2016 to March 2017. Patients with thyroid nodules with echographic criteria (TI-RADS 4,5
\end{abstract}

I Médico endocrinóloga. Servicio de Endocrinología, Hospital Nacional Arzobispo Loayza, Lima.

2 Médico endocrinólogo. Universidad Peruana Cayetano Heredia. Servicio de Endocrinología, Hospital Nacional Arzobispo Loayza, Lima.

3 Miembro del Centro de Excelencia en Enfermedades Crónicas (Cronicas), Universidad Peruana Cayetano Heredia. and 6) and cytological malignancy (Bethesda IV, V and VI) were referred to the head and neck surgery service. A total of 27 patients completed their clinical, ultrasound and cytohistological study. Results: From the 27 thyroidectomized patients, $70,37 \%$ had a malignant thyroid neoplasia, the majority was female patients over 40 year-old. The most frequent malignancy was papillary thyroid carcinoma; $68,42 \%$ of nodules were greater than $10 \mathrm{~mm}$. The majority of patients with nodule TI-RADS 4-5 and Bethesda IV, V and VI were histologically reported as malignant thyroid neoplasm; $75 \%$ of this patients were euthyroid. Conclusion: The correct categorization of the nodules according to their sonographic characteristics (TI-RADS Classification) allows the identification of patients who will require a biopsy in a timely manner. In this study it was found that the majority of patients with malignant thyroid neoplasia corresponded to nodules $\geq 10 \mathrm{~mm}$ with TI-RADS $4 b$ y 5 ultrasound criteria, and Bethesda $V$ and VI cytological criteria, mostly euthyroid. Papillary carcinoma is affirmed as the most frequent thyroid neoplasia.

Key words: Thyroid nodules, ultrasound, cytology, Bethesda, thyroid cancer. 


\section{INTRODUCCIÓN}

Los nódulos tiroideos (NT) son masas localizadas de consistencia generalmente firme que pueden distinguirse del resto del parénquima de la glándula. ${ }^{1} \mathrm{Su}$ incidencia es más alta con la edad y son ocho veces más frecuentes en mujeres que en hombres; asimismo, son frecuentes en personas provenientes de áreas con deficiencia de yodo. Por otro lado, la posibilidad de malignidad es dos veces mayores en hombres que en mujeres.

Los estudios han revelado una prevalencia de NT a la palpación del $3 \%$ al $7 \%$ en la población general, y por ultrasonido de $20 \%$ a $76 \%$. Los datos en autopsia reportan una prevalencia de $8 \%$ a $65 \% .{ }^{2-4}$ Los NT nuevos aparecen a un ritmo de $0,1 \%$ al año, multiplicándose por 20 en casos de irradiación de cabeza, cuello o tórax. ${ }^{5}$ Aunque la mayoría de los NT son benignos, la importancia de su estudio radica en la necesidad de descartar el cáncer de tiroides, el cual se encuentre entre el $4,0 \%$ al $6,5 \%$ de todos los NT. ${ }^{6}$

El ultrasonido (US) de alta resolución (transductor de $7 \mathrm{a} 15 \mathrm{MHz}$ ) es el método más sensible para detectar lesiones tiroideas, permite medir sus dimensiones, identificar su estructura y evaluar los cambios del parénquima. El US asociado a Doppler proporciona además información acerca del aumento de la vascularidad y la presencia de cortocircuitos arteriovenosos. ${ }^{1,7} \mathrm{Su}$ rendimiento en la detección de malignidad depende, entre otros factores, del tipo histológico específico del cáncer; así, mientras en las neoplasias no foliculares la sensibilidad alcanza el $86,5 \%$ y la especificidad un $92,3 \%$, en los foliculares el rendimiento cae a $18,2 \%$ en sensibilidad y a $88,7 \%$ de especificidad. $^{8}$

El sistema de clasificación TI-RADS, basada en la ecogenicidad, calcificaciones, vascularización y estructura del NT por ultrasonido, se ha diseñado con el fin de unificar criterios ultrasonográficos que permitan identificar a los NT que deben ser evaluados por la biopsia por aspiración con aguja fina (BAAF) ante la posibilidad de malignidad. ${ }^{9}$

La BAAF constituye actualmente el método de elección para el diagnóstico de la patología nodular tiroidea. ${ }^{10}$ Un promedio de $15 \%$ a $20 \%$ de los estudios puede ser inadecuados para formular diagnóstico por escasez o mala calidad del material; sin embargo, si el estudio es realizado por un operador experimentado y evaluado por un patólogo capacitado, brinda información de utilidad para el posterior manejo y abordaje de la lesión tiroidea. ${ }^{11}$ Numerosos estudios han reportado una sensibilidad de $65 \%$ a $100 \%$ y una especificidad de $70 \%$ a $100 \% .{ }^{12} \mathrm{Su}$ uso ha permitido disminuir el número de intervenciones quirúrgicas innecesarias. Dentro de sus ventajas, se puede destacar que es poco invasivo, económico, rápido y sencillo de realizar, además de presentar un riesgo de complicaciones mínimas. El apoyo del ultrasonido durante el procedimiento, constituye un valioso soporte durante la BAAF, necesario en lesiones no palpables y de utilidad en la caracterización de lesiones palpables. ${ }^{13}$

Por todo lo expuesto, en el presente estudio se buscó describir las características clínicas, ecográficas, citológicas (obtenido mediante el BAAF) e histopatológicas de los NT con sospecha de malignidad en pacientes atendidos en el área de ecografía del servicio de endocrinología de un hospital público.

\section{MATERIAL Y MÉTODOS}

Se realizó un estudio descriptivo y prospectivo de los pacientes con NT que acudieron al área de ecografía del servicio de endocrinología del Hospital Nacional Arzobispo Loayza, Lima, durante el periodo marzo de 2016 a marzo de 2017. Luego de un año de seguimiento, se incluyó a 27 pacientes con estudio clínico, ecográfico y citohistológico completos, sin importar sexo o edad. Se excluyó a los pacientes con diagnóstico de NT sin estudios completos.

\section{Procedimiento y técnicas}

Se utilizó un ecógrafo con transductor lineal de 10 $\mathrm{MHz}$ dotado con modo Doppler color. Se indicó el estudio citológico guiado por ecografía de la glándula tiroides a los nódulos sólidos de $10 \mathrm{~mm}$ a más y a las lesiones sospechosas de malignidad: nódulos de bordes irregulares, márgenes infiltrativos, presencia de microcalcificaciones, aumento de la vascularización intranodular y/o presencia de adenomegalias cervicales anormales.

Se categorizaron todos los NT bajo el sistema de clasificación TI-RADS, se consideraron a las categorías 4,5 y 6 con indicación para BAAF guiada por ecografía.

\section{Técnica de la citología del tiroides o biopsia por aspiración con aguja fina (BAAF)}

Previo a la aceptación del consentimiento informado, se inició con la exploración de la tiroides para identificar el nódulo. Luego de una asepsia local con alcohol, se 
procedió a punzar con la guía del ultrasonido; para esto se utilizó una jeringa descartable de $20 \mathrm{~mL}$, con aguja $21 \mathrm{G}$. Se aspiró por 5 a $10 \mathrm{~s}$, recorriendo el NT. La muestra obtenida fue extendida en seis portaobjetos identificados; luego, fijados por 10 a $15 \mathrm{~min}$, con alcohol al $96 \%$, y se los deja secar.

La lectura de las láminas estuvo cargo de un médico patólogo del hospital.

\section{Indicación quirúrgica}

Los resultados de citología tras la BAAF se informaron según la clasificación Bethesda.

Los pacientes que presentaron un reporte citológico Bethesda IV, V y VI y/o presentaron signos de invasión local como disfagia, disfonía o disnea fueron referidos al servicio de Cabeza y Cuello para su intervención quirúrgica.

\section{Técnica de estudio de la pieza quirúrgica de la tiroides}

Las piezas de biopsia fueron deshidratadas en formol al $10 \%$ y fijadas en parafina con cortes de $3 \mathrm{~mm}$. Los cortes histológicos fueron evaluados por un médico patólogo.

\section{Definición de variables}

Las variables de interés del estudio fueron malignidad y benignidad. Malignidad definida por el estudio microscópico cuando se encontraba cáncer papilar, cáncer folicular, cáncer anaplásico o cáncer medular. Benignidad definida por estudio microscópico cuando se encontraba hiperplasia multinodular o adenoma folicular.

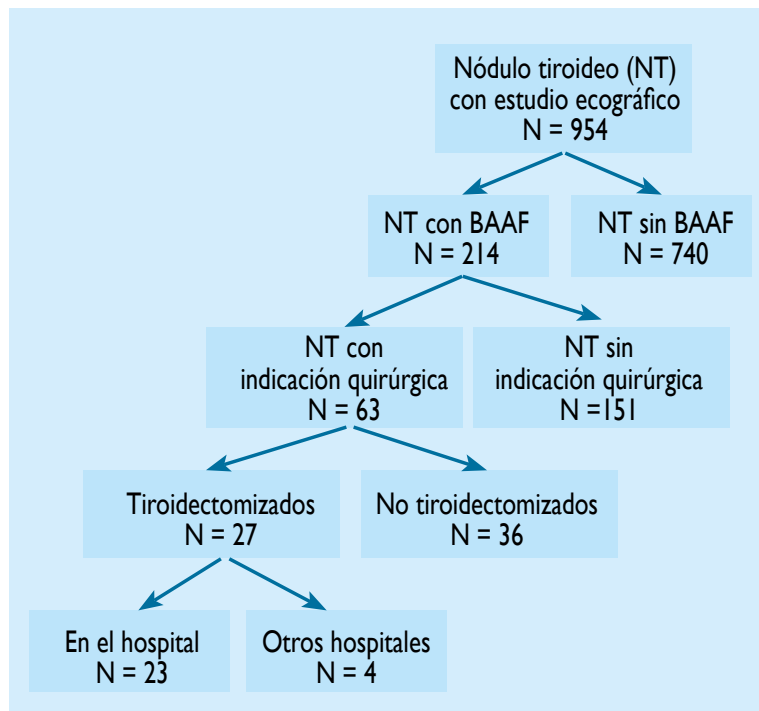

Figura I. Diagrama de flujo de la población de estudio.
Tabla I.Total de los pacientes con BAAF, según clasificación Bethesda $(T=214)$

\begin{tabular}{lrr}
\hline Clasificación de Bethesda & N & $\%$ \\
\hline- I & 46 & 21,5 \\
- II & 106 & 49,5 \\
- III & 10 & 4,7 \\
- IV & 14 & 6,5 \\
- V & 11 & 5,1 \\
- VI & 27 & 12,6 \\
\hline
\end{tabular}

Las clasificaciones de TI-RADS (1, 2, 3, 4a, 4b, 5, 6) y Bethesda (I, II, III, IV, V, VI) fueron otras variables de interés relacionadas al estudio ecográfico y citológico del NT, respectivamente. Anexos 1 y 2.

\section{Plan de análisis}

Los resultados se resumieron en frecuencias absolutas y porcentuales. Para variables continuas se determinaron medidas de tendencia central: media, desviación estándar, mediana, valores mínimos y máximos.

Los datos obtenidos fueron introducidos en una base de datos (Excel 2016) a partir de la cual fueron procesados estadísticamente, mediante el programa Stata versión 15.

\section{RESULTADOS}

De los 954 pacientes a los que se les realizó ecografía tiroidea, 214 requirieron BAAF y de ellos, 63 fueron referidos para intervención quirúrgica (NT con clasificación Bethesda III, IV, V y VI) (Tabla 1) Sin embargo, solo 27 pacientes completaron su estudio histológico tras la tiroidectomía (Figura 1). En 19 $(70,4 \%)$ de ellos, se confirmó el diagnóstico de neoplasia maligna en la anatomía patológica.

Tabla 2. Características epidemiológicas, según resultado histológico

\begin{tabular}{|c|c|c|c|c|}
\hline & \multicolumn{2}{|c|}{ Benigno $(n=8)$} & \multicolumn{2}{|c|}{ Maligno $(n=19)$} \\
\hline & $n$ & $\%$ & $n$ & $\%$ \\
\hline \multicolumn{5}{|l|}{ - Sexo } \\
\hline - Masculino & 0 & 0 & 3 & 100,00 \\
\hline - Femenino & 8 & 33,33 & 16 & 66,67 \\
\hline \multicolumn{5}{|l|}{ - Edad (años) } \\
\hline - Menor de 18 & 0 & 0 & 1 & 100,00 \\
\hline - De 18 a 30 años & 2 & 28,57 & 5 & 71,43 \\
\hline - De 31 a 40 años & 0 & 0 & 3 & 100,00 \\
\hline - Mayor de 40 años & 6 & 37,50 & 10 & 62,50 \\
\hline
\end{tabular}


Tabla 3. Resultado histológico, según función tiroidea y tamaño del nódulo

\begin{tabular}{|c|c|c|c|c|}
\hline \multirow[t]{2}{*}{ Función tiroidea } & \multicolumn{2}{|c|}{$\begin{array}{l}\text { Benigno } \\
(\mathrm{n}=8)\end{array}$} & \multicolumn{2}{|c|}{$\begin{array}{l}\text { Maligno } \\
(n=19)\end{array}$} \\
\hline & $\bar{n}$ & $\%$ & $\bar{n}$ & $\%$ \\
\hline - Hipertiroidismo & 2 & 50,00 & 2 & 50,00 \\
\hline - Hipotiroidismo & I & 33,33 & 2 & 66,66 \\
\hline - Eutiroideo & 5 & 25,00 & 15 & 75,00 \\
\hline \multicolumn{5}{|c|}{ - $\quad$ Tamaño del nódulo $(\mathrm{mm})$} \\
\hline - Menor de 10 & 0 & 0 & 6 & 100,00 \\
\hline & 8 & 38,10 & 13 & 61,90 \\
\hline
\end{tabular}

El $66,7 \%$ de pacientes mujeres y el $100 \%$ de varones presentaron el diagnóstico de carcinoma tiroideo, de ellos el 59,3\% era mayor de 40 años (Tabla 2).

Según las características clínicas, $75 \%$ de pacientes con carcinoma de tiroides presentaron función tiroidea normal (eutiroideos) y $61,9 \%$ tenían NT con un diámetro de 10 o más mm. Todos los NT menores de $10 \mathrm{~mm}$ fueron neoplasias malignas de tiroides (Tabla 3).

Según las características ecográficas del NT, 57,14\% de pacientes TI-RADS 4b y $100 \%$ de TI-RADS 5 tuvieron carcinoma de tiroides por histopatología (Tabla 4).

Según las características citológicas del NT, 33,3\% de NT con clasificación Bethesda IV y 75\% de los NT con clasificación Bethesda $\mathrm{V}$ confirmaron el diagnóstico de neoplasia maligna (Tabla 5).

La Tabla 6 describe los resultados histológicos de los pacientes tiroidectomizados, donde se observa que el $66,7 \%$ correspondió a carcinoma de tiroides variante

\begin{tabular}{lrrrrr} 
Tabla 4. Características ecográficas según resultado histológico \\
\hline Clasificación TI-RADS & \multicolumn{2}{c}{$\begin{array}{c}\text { Benigno } \\
(\mathrm{n}=8)\end{array}$} & & \multicolumn{2}{c}{$\begin{array}{c}\text { Maligno } \\
(\mathrm{n}=19)\end{array}$} \\
\cline { 2 - 6 } & $\mathrm{n}$ & $\%$ & & $\mathrm{n}$ & $\%$ \\
\hline-1 & 0 & 0 & & 0 & 0 \\
-2 & 0 & 0 & & 1 & 100,00 \\
-3 & 2 & 40,00 & & 3 & 60,00 \\
$-4 a$ & 2 & 50,00 & & 50,00 \\
$-4 \mathrm{~b}$ & 4 & 36,36 & 7 & 57,14 \\
-5 & 0 & 0 & 6 & 100,00 \\
-6 & 0 & 0 & 0 & 0 \\
\hline
\end{tabular}

\begin{tabular}{lrrrrr} 
Tabla 5. Características citológicos, según resultado histológico \\
\hline Clasificación Bethesda & \multicolumn{2}{c}{$\begin{array}{c}\text { Benigno } \\
(n=8)\end{array}$} & & \multicolumn{2}{c}{$\begin{array}{c}\text { Maligno } \\
(n=19)\end{array}$} \\
\cline { 2 - 6 } & $n$ & $\%$ & & $n$ & $\%$ \\
\hline - I & 0 & 0 & & 0 \\
- II & 0 & 0 & & 100,0 \\
- III & 1 & 100,0 & 0 & 0 \\
- IV & 6 & 66,7 & 3 & 33,3 \\
- V & 1 & 25,0 & 3 & 75,0 \\
- VI & 0 & 0 & 12 & 100,0 \\
\hline
\end{tabular}

clásica; y, el 17,6\% de los pacientes con histología maligna fue reportado también con tiroiditis linfocítica crónica.

\section{DISCUSIÓN}

El presente estudio encontró que la mayor parte de pacientes que acudió a ecografía estuvo constituida por mujeres, con una edad promedio de $41 \pm 13$ años. Vargas y col. también encontraron que la mayoría de participantes fueron mujeres, con edad media de 57 años. ${ }^{15}$

El ultrasonido (US) es el método de estudio de elección para el abordaje inicial de los NT. ${ }^{14}$ Actualmente, el uso de la ecografía tiroidea es indispensable para la caracterización de los NT así como método para guiar las biopsias de los mismos. La decisión para la realización de biopsias se basó en los criterios clínicos (NT $\geq 10 \mathrm{~mm}$ y/o síntomas mecánicos) y por signos ecográficos, principalmente. ${ }^{16}$

\begin{tabular}{lrc}
$\begin{array}{l}\text { Tabla 6. Resultados histopatológicos de los pacientes } \\
\text { tiroidectomizados ( }=27)\end{array}$ & & \\
\hline & $\mathrm{N}$ & $\%$ \\
\hline & 5 & 18,52 \\
- Hiperplasia nodular & 3 & $11,1 \mathrm{I}$ \\
- Adenoma folicular & 18 & 66,67 \\
- Carcinoma papilar variante clásica & $\mathrm{I}$ & 3,70 \\
- Carcinoma papilar variante folicular & 0 & 0,00 \\
- Carcinoma folicular & 0 & 0,00 \\
- Carcinoma anaplásico & 0 & 0,00 \\
- Carcinoma medular & & \\
- Carcinoma con tiroiditis linfocítica crónica (N = I7) & 5 & 26,32 \\
- Sí & 14 & 7,68 \\
\hline - No & &
\end{tabular}




\section{Anexo I. Clasificación TI-RADS (I7,20-22)}

\begin{tabular}{|c|c|}
\hline - TI-RADS I & Tiroides normal. Ninguna lesión focal. ( $0 \%$ de riesgo de malignidad) \\
\hline - TI-RADS 2 & $\begin{array}{l}\text { Lesión benigna, incluye: (< } 5 \text { \% de riesgo de malignidad) } \\
\text { - Quiste coloide típico, áreas anecoicas y manchas hiperecogénicas. } \\
\text { - Nódulo mixto, no encapsulado con apariencia de malla, dado por áreas sólidas isoecoicas y manchas hiperecoicas. } \\
\text { - Nódulo mixto, no encapsulado, isoecoico, con manchas hiperecoicas y vascularizado. }\end{array}$ \\
\hline - TI-RADS 3 & $\begin{array}{l}\text { Nódulo probablemente benigno, incluye: nódulo hiper, iso o hipoecoico, parcialmente encapsulado con } \\
\text { vascularización periférica, con aspecto sugestivo de tiroiditis de Hashimoto. }\end{array}$ \\
\hline - TI-RADS 4 & \\
\hline$-4 A$ & $\begin{array}{l}\text { Nódulo indeterminado, incluye: (5-10\% de riesgo de malignidad) } \\
\text { - Patrón neoplásico simple, nódulo sólido o mixto hiper, iso o hipoecoico sin calcificaciones, rodeado por fina cápsula. } \\
\text { - Patrón de Quervain, lesión hipoecoica con bordes mal definidos sin calcificaciones. } \\
\text { - Patrón neoplásico sospechoso; nódulo hiper, iso o hipoecoico, hipervascularizado, rodeado por cápsula gruesa y } \\
\text { con calcificaciones. }\end{array}$ \\
\hline - 4B: & $\begin{array}{l}\text { Nódulo sospechoso para malignidad, incluye: (10-50\% de riesgo de malignidad) } \\
\text { - Nódulo sólido hipoecoico, no encapsulado, con forma y márgenes mal definidos, vascularizado y con o sin calcificaciones. }\end{array}$ \\
\hline - TI-RADS 5 & $\begin{array}{l}\text { Nódulo compatible con malignidad, (>85\% de riesgo de malignidad) incluye: } \\
\text { nódulo sólido, no encapsulado, isoecoico o hipoecoico, hipervascularizado y con múltiples calcificaciones periféricas. }\end{array}$ \\
\hline - TI-RADS 6 & $\begin{array}{l}\text { Nódulo maligno, incluye: nódulo mixto, hipoecoico, hipervascularizado y no encapsulado } \\
\text { con o sin calcificaciones y sin manchas hiperecoicas, que ya han sido confirmados por biopsia previa. }\end{array}$ \\
\hline
\end{tabular}

En este estudio se encontró que $57,14 \%$ de pacientes TI-RADS $4 \mathrm{~b}$ y el $100 \%$ de TI-RADS 5 presentaron histopatología de malignidad. Datos similares a los mencionados en el estudio de Zhuang y col., ${ }^{19}$ en donde se reportó $75 \%$ de malignidad en la clasificación TIRADS 4 y $98 \%$ en la clasificación TI-RADS 5. En el estudio prospectivo de Horvath y col. también se confirmó una patología maligna de tiroides en los nódulos descritos con clasificación TI-RADS 4 y 5 $\left(76,13 \%\right.$ y 98,85 , respectivamente)..$^{17}$

Del total de pacientes, el 21,49\% (de 214 biopsias, 46 fueron Bethesda I) se informaron como Bethesda I (no diagnóstico/insuficiente), cifra que superó a la reportada en la literatura mundial en la que se espera que esto ocurra entre $7 \%$ y $10 \% .^{14,18}$ Esta cifra permite evaluar e identificar los posibles errores en el procedimiento y lectura de las biopsias. De los 27 pacientes tiroidectomizados, en más de la mitad se confirmó el diagnóstico de neoplasia maligna; y, de estos, 94,7\% fueron informados como TI-RADS 3,4 y $5 .^{19}$

La BAAF constituye el método idóneo para la selección de pacientes que requieren intervención quirúrgica, considerando a los NT malignos o sospechosos de malignidad para estos casos.

En todos los casos reportados como Bethesda VI (carcinoma papilar de tiroides) se confirmó el diagnóstico
Anexo 2. El sistema Bethesda para reportar la citopatología de tiroides: categorías diagnósticas recomendadas. $15,23,24$

- I. No diagnostico o insatisfactoria:
a. Fluido quístico
b. Espécimen acelular
c. Otros (sangre, artefactos, etc.)

- II. Benigno
a. Consistente con nódulo folicular benigno
b. Consistente con tiroiditis linfocitaria, en el contexto clínico adecuado
c. Consistente de tiroiditis granulomatosa
d. Otros

- III. Atipia de significado incierto o lesión folicular de significado indeterminado

- IV. Neoplasia folicular o sospechoso de neoplasia folicular a. Especifica si existe células de Hurthle

- V. Sospechoso de malignidad:
a. Sospechoso de carcinoma papilar
b. Sospechoso de carcinoma medular
c. Sospechoso de carcinoma metastásico
d. Sospechoso de linfoma
e. Otros

- VI. Maligno
a. Carcinoma papilar de tiroides
b. Carcinoma pobremente diferenciado
c. Carcinoma medular de tiroides
d. Carcinoma indiferenciado (anaplásico)
e. Carcinoma de células escamosas
f. Carcinoma con características mixtas
g. Carcinoma metastásico
h. Carcinoma no Hodgkin
i. Otros 
en el estudio histológico de la pieza operatoria, lo que presume la alta precisión del estudio citológico en el diagnóstico de carcinoma papilar.

El NT benigno más común es la hiperplasia nodular, mientras que la principal neoplasia maligna de tiroides es el cáncer papilar $(75 \%),{ }^{16}$ ambos datos se repitieron en este estudio.

Debido a que algunos de los pacientes no llegaron a ser intervenidos quirúrgicamente en nuestro hospital, no se pudo correlacionar todo el universo de los diagnósticos citológicos con los resultados histológicos.

En conclusión, solo 27 pacientes lograron ser intervenidos quirúrgicamente durante un año de seguimiento. La mayoría de ellos fue del sexo femenino, con una edad promedio de 40,8 años de edad. Los pacientes con neoplasia maligna de tiroides presentaron NT $\geq 10 \mathrm{~mm}$ con criterios ecográficos TI-RADS 4 b y 5 , y criterios citológicos Bethesda V y VI, clínicamente con función tiroidea normal.

Finalmentela mayor parte de pacientes tiroidectomizados presentaron el diagnóstico histológico de carcinoma papilar de tiroides variante clásica. Ante los resultados encontrados en el presente estudio, se puede mencionar a la BAAF como un procedimiento útil para definir la indicación quirúrgica de un NT sospechoso de malignidad, por lo que sería recomendable su uso en pacientes con características ecográficas específicas según la clasificación TI-RADS. Así mismo, cabe reafirmar que el éxito del diagnóstico dependerá de la experiencia del médico a cargo de la ecografía, de la toma de muestra por biopsia y la lectura de la misma.

Agradecimientos: A las doctoras Isabel Yoshida Honma, Guiselle Gutierrez Guerra y Zenaida Lozano Miranda, por el apoyo brindado en las lecturas minuciosas de las muestras.

\section{REFERENCIAS BIBLIOGRÁFICAS}

I. Rivera-Moscoso R, Hernández-jiménez S, Ochoa-Sosa A, RodríguezCarranza S, Torres-Ambriz P. Diagnóstico y tratamiento del nódulo tiroideo. Posición de la Sociedad Mexicana de Nutrición y Endocrinología, A.C. Rev Endocrinol Nutr. 2010;18(I):34-50.

2. Gharib H, Papini E, Paschke R, Duick D, Valcavi R, Hegedüs $L$, et al. American Association of Clinical Endocrinologists, Associazione Medici Endocrinologi and European Thyroid Association. Medical guidelines for clinical practice for the diagnosis and management of thyroid nodules. Endocr Pract. 2010;16(Suppl I): I-43.

3. Reading CC, Charboneau WJ, Hay ID, Sebo TJ. Sonography of thyroid nodules:A "classic pattern" diagnostic approach. Ultrasound Quarterly. 2005;2I(3):157-65.

4. Guía práctica para el diagnóstico y tratamiento del nódulo tiroideo. URL disponible en: https://docplayer.es/2058662-Guia-practica-para-eldiagnostico-y-tratamiento-del- nodulo-tiroideo.html

5. Tan GH. Thyroid incidentalomas: management approaches to nonpalpable nodules discovered incidentally on thyroid imaging. Ann Intern Med. 1997;126(3):226.
6. Haugen BR, Alexander EK, Bible KC, Doherty GM, Mandel SJ, Nikiforov YE, et al. 2015 American Thyroid Association Management Guidelines for Adult patients with thyroid nodules and differentiated thyroid cancer: The American Thyroid Association Guidelines Task Force on Thyroid Nodules and Differentiated Thyroid Cancer.Thyroid. 2016;26(I):I-I33.

7. Desser TS, Kamaya A. Ultrasound of thyroid nodules. Neuroimaging Clin N Am. 2008; 18(3):463-78.

8. Horvath E, Majlis S, Rossi R, Franco C, Niedmann JP, Castro A, et al. An ultrasonogram reporting system for thyroid nodules stratifying cancer risk for clinical management.J Clin Endocrinol Metab. 2009;94:I748-5I.

9. Kim DW, Lee EJ, Kim SH, Kim TH, Lee SH, Kim DH, et al. Ultrasoundguided fine- needle aspiration biopsy of thyroid nodules: comparison in efficacy according to nodule size. Thyroid. 2009;19(I):27-31.

10. Khalid AN, Hollenbeak CS, Quraishi SA, Fan CY, Stack BC. The costeffectiveness of iodine I3I scintigraphy, ultrasonography, and fine-needle aspiration biopsy in the initial diagnosis of solitary thyroid nodules. Arch Otolaryngol Neck Surg. 2006; I 32 (3):244.

II. Ibarra A, Wash A, Matamala P, O'Brian A. Diagnóstico citológico en patología tiroidea: estudio bajo ultrasonido con asistencia del citopatólogo. Rev Médica Clínica Las Condes. 20I I;22(4):508-I I.

12. Legal Balmaceda E, Sosa Ramírez C. Fine needle aspiration (FNA) to clasify thyroid nodules. Cirugía paraguaya. 2018;42(I):24-7.

13. Gharib H, Papini E.Thyroid nodules: clinical importance, assessment, and treatment. Endocrinol Metab Clin North Am. 2007;36(3):707-35.

14. Nachiappan AC, Metwalli ZA, Hailey BS, Patel RA, Ostrowski ML,Wynne DM. The thyroid: review of imaging features and biopsy techniques with radiologic- pathologic correlation. RadioGraphics. 2014;34(2):276-93.

15. Vargas-Uricoechea H, Meza-Cabrera I,Herrera-ChaparroJ.Concordance between the TIRADS ultrasound criteria and the Bethesda cytology criteria on the nontoxic thyroid nodule. Thyroid Res. 2017;10:1.

16. Herrera R, Lozano H, Perez J, et al. Biopsia por aspiración con aguja fina guiada por ultrasonido y correlación citopatológica de nódulos tiroideos en un hospital privado de México.Acta médica grupo ángeles. 2015; I3(I)

17. Horvath E, Silva CF, Majlis S, Rodriguez I, Skoknic V, Castro A, et al. Prospective validation of the ultrasound based TIRADS (Thyroid Imaging Reporting And Data System) classification: results in surgically resected thyroid nodules. Eur Radiol. 2017;27(6):2619-28.

18. Periakaruppan G, Seshadri KG, Krishna GV, Mandava R, Sai PV, Rajendiran S. Correlation between ultrasound-based TIRADS and Bethesda system for reporting thyroid-cytopathology: 2-year experience at a tertiary care center in India. Indian J Endocrinol Metab. 2018;22(5):65I.

19. Zhuang Y, Li C, Hua Z, Chen K, Lin JL.A novel TIRADS of US classification. Biomed Eng Online. 2018;17(I):82.

20. Vista de Clasificación TIRADS - una herramienta útil en la selección de nódulos tiroídeos que requieren punción diagnóstica [Internet]. [citado 8 de abril de 2019]. URL disponible en: http://contactocientifico.alemana. cl/ojs/index.php/cc/article/view/8I/83

21. Fernández Sánchez J. Clasificación TI-RADS de los nódulos tiroideos en base a una escala de puntuación modificada con respecto a los criterios ecográficos de malignidad. Rev Argent Radiol. 2014;78(3): 138-48.

22. Frates MC, Benson CB, Charboneau JW, Cibas ES, Clark OH, Coleman BG, et al. Management of thyroid nodules detected at US: Society of Radiologists in Ultrasound Consensus Conference Statement. Radiology. 2005;237(3):794-800.

23. Romero-Rojas A, Melo-Uribe MA. Implementación del Sistema Bethesda para el informe de citología aspirativa de tiroides con seguimiento histopatológico: experiencia en un centro de tratamiento de cáncer. Rev Colomb Cancerol. 2014; 18(I):3-7.

24. Hoyos AV, de Santamaría JS, Franceschi IJ, García AL, Rodríguez P, Varela G, et al. Consenso médico de biopsia por aspiración con aguja fina de tiroides. Reporte de citología tiroidea basado en el sistema de Bethesda: 10.

\section{CoRRESPONDENCIA: Liz Kathia Mendoza Montoya} liz.mendoza.m@upch.pe

CONFLICTO DE INTERESES: Ninguno, según los autores.

FINANCIAMIENTO: Por los autores.

FECHA DE RECEPCIÓN: I I de abril de 2019.

FECHA DE ACEPTACIÓN: 20 de mayo de 2019. 